\title{
Quaderni
}

QUADERNI Communication, technologies, pouvoir

\section{Judith Bonnin, Les voyages de François Mitterrand. Le PS et le monde (1971-1981)}

\section{Rémi Dalisson}

\section{(2) OpenEdition \\ 12 Journals}

Édition électronique

URL : http://journals.openedition.org/quaderni/990

DOI : 10.4000/quaderni.990

ISSN : 2105-2956

Éditeur

Les éditions de la Maison des sciences de l'Homme

\section{Édition imprimée}

Date de publication : 5 mai 2016

Pagination : 131-133

\section{Référence électronique}

Rémi Dalisson, "Judith Bonnin, Les voyages de François Mitterrand. Le PS et le monde (1971-1981) », Quaderni [En ligne], 90 | Printemps 2016, mis en ligne le 05 mai 2016, consulté le 22 septembre 2020.

URL : http://journals.openedition.org/quaderni/990 ; DOI : https://doi.org/10.4000/quaderni.990 


\section{Compte rendu}

\section{Les voyages de François Mitterrand. Le PS et le monde (1971-1981)}

\section{Judith Bonnin}

Rennes, PUR, 2014

par Rémi Dalisson

Université de Rouen

L'ouvrage de Judith Bonnin est remarquable à plus d'un titre. Il est d'abord le fruit d'un « simple » mémoire de master qui fleure bon le doctorat et montre tout le talent de cette jeune historienne qui prépare actuellement sa thèse. Il s'inscrit en outre dans la meilleure veine d'une histoire culturelle du politique, et plus particulièrement dans l'histoire des rituels et symboliques politiques qui révèlent « l'infra-politique enfoui », selon le joli mot de JeanFrançois Sirinelli.

Enfin, c'est un beau travail d'une historienne qui puise aux meilleures sources qu'elle n'omet pas de critiquer (en partie seulement d'ailleurs). Il s'agit de la presse généraliste (Le Monde, le Nouvel observateur) et militante (notamment la Nouvelle revue socialiste), des archives de première main du même parti et de l'État, des biographies de personnalités socialistes (Attali, Poperen...) et de l'histoire orale à travers des interviews (Estier, le Garrec). Elle n'oublie pas les archives iconographiques et télévisuelles de l'INA (que détestait tant Mitterrand à ses débuts), les ouvrages savants sur la pratique du voyage et les politiques symboliques et le regard de l'étranger. L'ensemble du travail est exposé à travers un plan clair et lisible dans un style aussi agréable que précis.

L'étude des 88 voyages à l'étranger du futur président, souvent en Europe de l'Ouest (p. 243, carte p. 245), outre qu'elle permet de croiser toute une génération politique en (petite) partie encore active de nos jours, est fascinante. Aucun grand pays n'est écarté, qu'il soit capitaliste ou socialiste, aucun continent n'est oublié et les rencontres sont aussi variées que riches, y compris celles qui n'ont pas eu lieu, les «non-voyages » du premier 
chapitre. Cette « diplomatie partidaire », qui se professionnalise rapidement, a une triple fonction longtemps sous-estimée. Elle a d'abord une fonction purement politique : donner une stature internationale à François Mitterrand, en dépit de son image intérieure souvent raillée. Mais elle doit aussi le légitimer aux yeux de son propre parti, où les opposants sont nombreux, tout en renforçant sa stature devant l'opinion publique, chose que n'aimait guère faire le futur candidat. Cette partie (la première de l'ouvrage) montre bien les difficultés matérielles des socialistes d'alors pour bâtir des délégations (le rôle des bénévoles), financer les voyages (la problématique des cadeaux est intéressante) et définir ce que l'auteur appelle un « tourisme politique » qui doit être politiquement signifiant. C'est aussi le moment de l'ouverture culturelle au monde et l'on y retrouve, dans cet après mai 1968, toute la culture et la curiosité de Mitterrand «trop souvent contraint de renoncer [...] à presque tout ce qui ne constituait pas l'objet de notre démarche, la politique ». C'est alors un magnifique banc d'essai pour les joutes nationales autant qu'un moyen d'adouber certains compagnons (accompagnateurs, cadres...). Chaque aspect de ces voyages, parfois longs et fastidieux (une semaine dans l'URSS brejnévienne), devient alors un acte politique au sens le plus noble et signifiant du terme

Mais c'est la seconde fonction qui est la plus passionnante et l'auteur la démontre fort bien. Il s'agit de la création d'un réseau de sociabilité politique pluriel de premier ordre qui prépare les victoires futures, rassemble le parti, l'ouvre au monde et révèle une véritable «vision du monde ». Les amitiés se nouent, les affinités se renforcent dans l'espace mondial (Garcia Marquez par exemple, mais aussi la fascination pour Brandt et Olof Palme) et dans le parti socialiste (le rôle de Deferre et Estier, la question Chevènement), des choix se dessinent qui se structurent autour d'un corpus de valeurs (les Droits de l'homme, l'anti-américanisme) qui fonde le parti en (re)conquête.

La troisième fonction découle de la précédente, c'est la fonction symbolique et diplomatique, la question géopolitique, cette « carte mentale » du parti de François Mitterrand d'avant le pouvoir. Car il y a bien un « imaginaire » (p. 236) des voyages internationaux dont le Chili et la Palestine seraient les deux axes. Se crée ainsi un internationalisme tiers-mondiste, un antiimpérialisme mâtiné d'anti-stalinisme que les réalités du pouvoir vont vite tempérer (voir Jean-Pierre Cot ou Claude Cheysson). On constate alors la radicalité de la vision du monde socialiste qui reflète bien cette fin de 
Trente Glorieuses, quand les idéologies n'avaient pas disparu et que la crise économique et sociale n'avait pas encore produit tous ses effets délétères, comme le repli sur soi.

Enfin, il faut nuancer le propos : ces voyages ne sont que ceux d'un chef de l'opposition, et leurs conséquences doivent être relativisées, ce que l'auteur aurait dû mieux préciser un peu plus (la conclusion aurait dû être plus fournie). C'est aussi une manière de diplomatie spectacle, de mise en scène qui relève de la modernité en politique qui n'empêche pas d'avoir une vision du monde très idéologisée à long terme. Surtout, c'est là que se forme la doctrine diplomatique du premier gouvernement socialiste dans un monde aujourd'hui largement disparu.

Au total, un ouvrage à la fois utile, savant (même s'il eut fallu un peu plus s'interroger sur les outils et concepts, y compris le statut de l'archive) qui donne envie d'en savoir plus sur le choc de cet avant pouvoir avec la réalité. Il confirme aussi tout l'intérêt de cet objet très politique qu'est le voyage présidentiel, aspect déjà abordé par Nicolas Mariot et d'autres (Sudhir Hazareesing, Juliette Glickman, Rémi Dalisson ou Olivier Ihl) pour des époques précédentes et à l'échelle nationale. 
